\title{
Using Theory to Reinterpret the Kinetics of Monofunctional Platinum Anticancer Drugs: Stacking Matters
}

\author{
Daniele Veclani,${ }^{\dagger}$ Andrea Melchior, ${ }^{* \dagger}$ Marilena Tolazzi, ${ }^{\dagger}$ and José P. Cerón-Carrasco**
}

'Dipartimento Politecnico di Ingegneria e Architettura (DPIA), Laboratori di Chimica, Università di Udine, via delle Scienze 9933100 Udine *Bioinformatics and High-Performance Computing Research Group (BIO-HPC), Universidad Católica San Antonio de Murcia (UCAM), Campus de los Jerónimos, 30107, Murcia, Spain.

\begin{abstract}
The monofunctional platinum drug phenanthriplatin (phenPt) blocks the replication of cancer cells even if it reacts with only one guanine base. However, there is still insufficient experimental data to improve its cytotoxicity and all previously proposed chemical modifications of the parent structure have resulted in a loss of activity. We use theoretical tools to illustrate the key steps in the biological mechanisms of phenPt - that is, its activation in water and subsequent attack on DNA. Our simulations suggest that the measured kinetic parameters, which are based on free nucleobases in solution, need to be reinterpreted because a self-assembled stacked reactive adduct is formed that is inaccessible in real DNA. The constants reported here will help guide future work in the synthesis of anticancer platinum drugs.
\end{abstract}

It was previously thought that only bifunctional platinum drugs, such as cisplatin [cisPt, cis- $\mathrm{Pt}\left(\mathrm{NH}_{3}\right)_{2} \mathrm{Cl}_{2}$ ], are of use in cancer chemotherapy, ${ }^{1}$ despite the fact that the efficacy of cisPt is limited by its severe side-effects and tumors may develop resistance during the first few months of treatment. ${ }^{2}$ Promising progress into the design of better cancer drugs has recently been made by Lippard and coworkers, ${ }^{3}$ who synthesized phenanthriplatin [phenPt, $\mathrm{Pt}\left(\mathrm{NH}_{3}\right)_{2}$ (phenanthridine) $\mathrm{Cl}^{+}$]. This molecule is active against 60 human cancer cell lines and has a higher cytotoxicity than cisPt. ${ }^{3}$ Figure 1 shows that cisPt-like drugs attack two DNA bases, inducing a severe distortion in the double-stranded structure, whereas phenPt is a monofunctional platinum drug and reacts with only one base. ${ }^{4}$ The DNA architecture is therefore initially retained after treatment with phenPt. The attached metallo-drug later twists the single strand of the DNA template at the replication stage, which switches on the cell suicide program. ${ }^{5}$ Non-targeted biomolecules (e.g., proteins rich in cysteine) are also damaged by both types of drug, which not only causes early inactivation, but also favors the development of resistance to treatment. ${ }^{6}$

The chemical reactions shown in Figure 1 need to be characterized before this novel phenPt-based metallodrug can be used clinically. The formation of covalent adducts of phenPt with isolated guanine and adenine moieties has previously been studied to determine the equilibrium and rate constants. ${ }^{7}$ These experiments suggested that the induced lesions are kinetically controlled, although it is unclear whether these results can be directly extrapolated to in vivo conditions. To help to resolve this key question, we assessed the reactivity of phenPt using density functional theory (DFT) calculations, a computational approach widely exploited in studying the reactivity of bifunctional metallo-drugs, ${ }^{8-12}$ but rarely used for monofunctional drugs such as phenPt. ${ }^{13}$ Our conclusions are based on B3LYP-D3 calculations implemented in Gaussian 16. ${ }^{14,15}$ All the quantum mechanical details are given in the Supporting Information (SI).

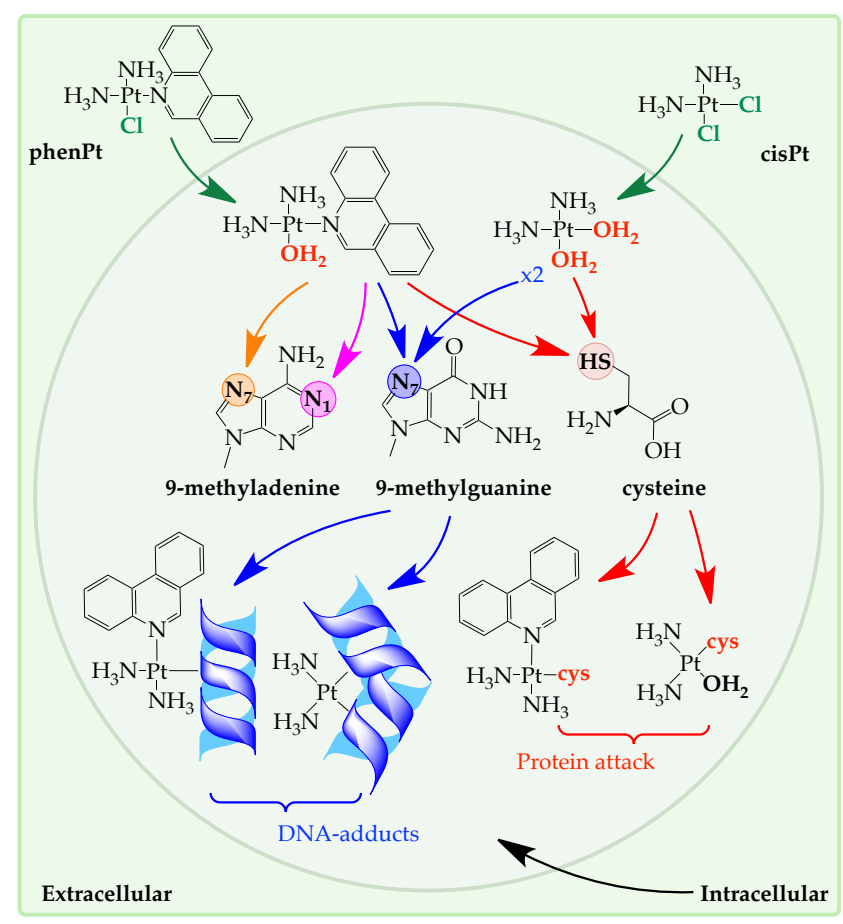

Figure 1. Schematic representation of the biological action of the cisplatin (cisPt) and phenanthriplatin (phenPt) metallo-drugs. From top to bottom: activation by hydrolysis; targeted reaction with DNA (modeled as 9-methyladenine and 9-methylguanine); and reaction with a cysteine (cys) residue present in proteins. The main products are associated with the attack on the N7 position in guanine (blue arrows) and to the sulfur in cysteine (red arrows), which lead to DNA adducts and an attack on proteins, respectively. 
The initial step in the computational strategy is to fully optimize all the species involved in the reaction, including the reagents $(\mathbf{R})$, the reactive adducts $(\mathbf{R A})$, the transition states (TS), the product adducts (PA) and the separated products $(\mathbf{P})$. These structures correspond to the critical points in the energetic profile of the reaction (see SI). Although experiments did not capture all these species, X-ray data are available for the isolated drug and the final products of the reaction with adenine and guanine and these data were used to validate our methodology. 5,16 phenPt can present as two isomers. Both forms were considered in this work, although all the values reported here are from isomer-M because we observed only a very small impact of the stereoisomers on the reactivity. Further details about the structure of these isomers are given in the SI and in ref. 16. The minimum energy structures perfectly match the deposited experimental structures ${ }^{7}$ with a deviation of $c .0 .03 \AA$ and $3^{\circ}$ for the bond lengths and angles, respectively. The agreement between the theoretical (Table S1) and experimental geometries confirms the reliability of our strategy and ensures the quality of the delivered predictions.

The reaction profile of phenPt corresponds to a mechanism starting with the hydrolysis of the leaving groups (chloride ligands marked in green at the top of Figure 1). Our calculations show that one water molecule enters the metal coordination sphere in similar way in both phenPt and cisPt. The incoming water molecule establishes a hydrogen bond with the ammonia ligand and a non-covalent bond that reorients the reactive atoms in the RA. This adduct subsequently yields to the rupture of the bond between platinum and the chloride ligand and the simultaneous formation of a metal-water bond (all structures given in the SI). The penta-coordinated nature of the TS is monitored by the single-imaginary vibrational mode, which corresponds to the bond formation/breaking process (Figure S2 and Table S7). The computed activation Gibbs free energy barrier and rate constant in Figure 2 $\left(\Delta G^{*}=24.2 \mathrm{kcal} \mathrm{mol}^{-1}\right.$ and $\left.k=1.1 \times 10^{-5} \mathrm{~s}^{-1}\right)$ are consistent with the experimentally recorded values. ${ }^{7}$ To extract a biological conclusion from these results, we need to compare the numerical outcome with the activation of cisPt, which remains the gold standard for cancer treatment. The calculations for cisPt $\left(\Delta G^{\ddagger}=24.0 \mathrm{kcal} \mathrm{mol}^{-1}\right.$ and $\left.k=1.6 \times 10^{-5} \mathrm{~s}^{-1}\right)$ are consistent with the literature value $^{17-20}$ and we therefore conclude that the presence of a phenanthridine ring does not alter the kinetics of the hydrolysis/activation step.

We assessed the critical step in the biological activity of cisPt and phenPt: the attack of the water-activated drugs on DNA. For consistency with previous work, ${ }^{7}$ the reactions were modeled with 9-methylguanine (at the N7 position) and 9-methyladenine (at the N1 and N7 positions). The energies are shown in Figure 2 and more details are presented in Figures S3 and S4. The water-phenPt complex interacts differently with the DNA purine bases. The reaction with guanine leads to a direct exchange of the inserted water molecule at the $\mathrm{N} 7$ position of the guanine. By contrast, the attack on the adenine base involves the transfer of a proton from the coordinated water molecule to the N7 position of the DNA base, an equilibrium previously reported for adenine, but not observed in guanine..$^{21,22}$ The proton transfer and the water $\rightarrow$ N7 equilibria increase the partial charge of the adenine moiety when the RA is formed. As a consequence, the reaction of

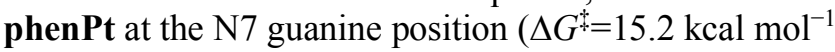
and $k=4.4 \times 10^{1} \mathrm{~s}^{-1}$ ) is preferred to the damage of adenine at either the $\mathrm{N} 7\left(\Delta G^{\sharp}=17.1 \mathrm{kcal} \mathrm{mol}^{-1}\right.$ and $k=1.8 \times$ $\left.10^{0} \mathrm{~s}^{-1}\right)$ or $\mathrm{N} 1\left(\Delta G^{*}=19.1 \mathrm{kcal} \mathrm{mol}^{-1}\right.$ and $k=6.2 \times 10^{-2}$ $\mathrm{s}^{-1}$ ) sites. The N7 adduct of both guanine and adenine leads to the most stable platination products, although the guanine product is kinetically favored. The stability of the induced damage by phenPt $\left(\Delta G=-4.2 \mathrm{kcal} \mathrm{mol}^{-1}\right.$ and $\left.K_{\text {eq }}=1.2 \times 10^{3}\right)$ and $\operatorname{cisPt}\left(\Delta G=-4.7 \mathrm{kcal} \mathrm{mol}^{-1}\right.$ and $K_{\text {eq }}$ $\left.=2.8 \times 10^{3}\right)$ drugs are similar.

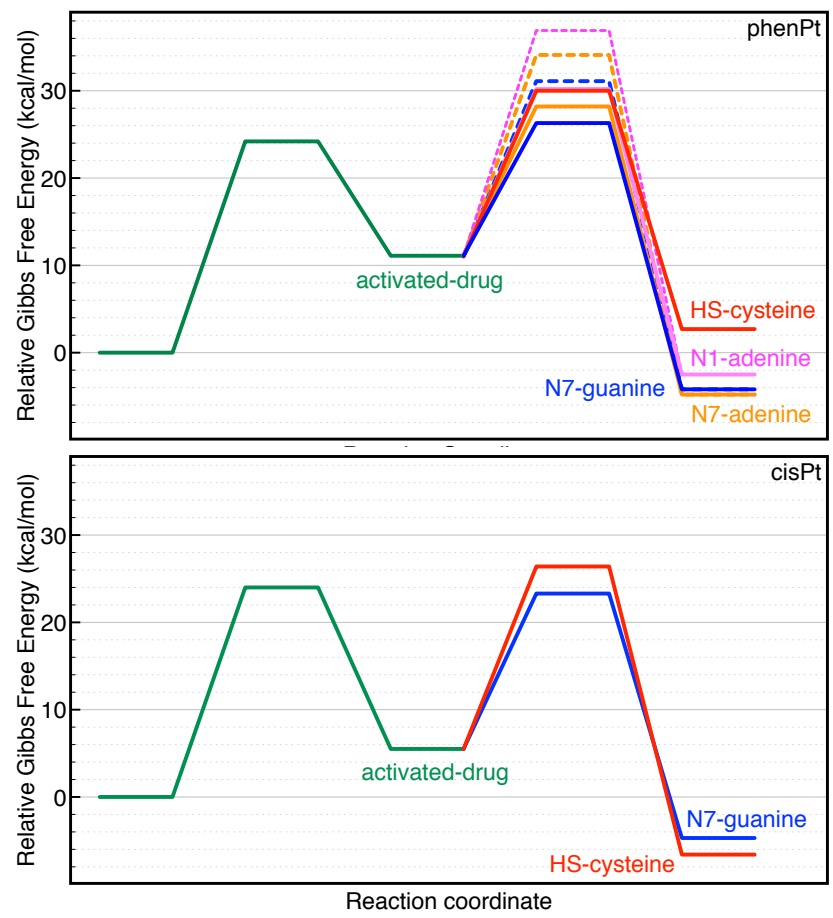

Figure 2. Free energy profiles for the reactions of phenPt (upper panel) and cisPt (lower panel): activation by hydrolysis (green); reaction at the N7 (orange) and N1 (pink) positions of 9-methyladenine; attack at the N7 position of the 9methylguanine (blue); and reaction with a cysteine (red). The alternative mechanism for phenPt via the self-assembled reactive adduct is indicated by dashed lines. The same energy scale is used in both panels. $O$ 

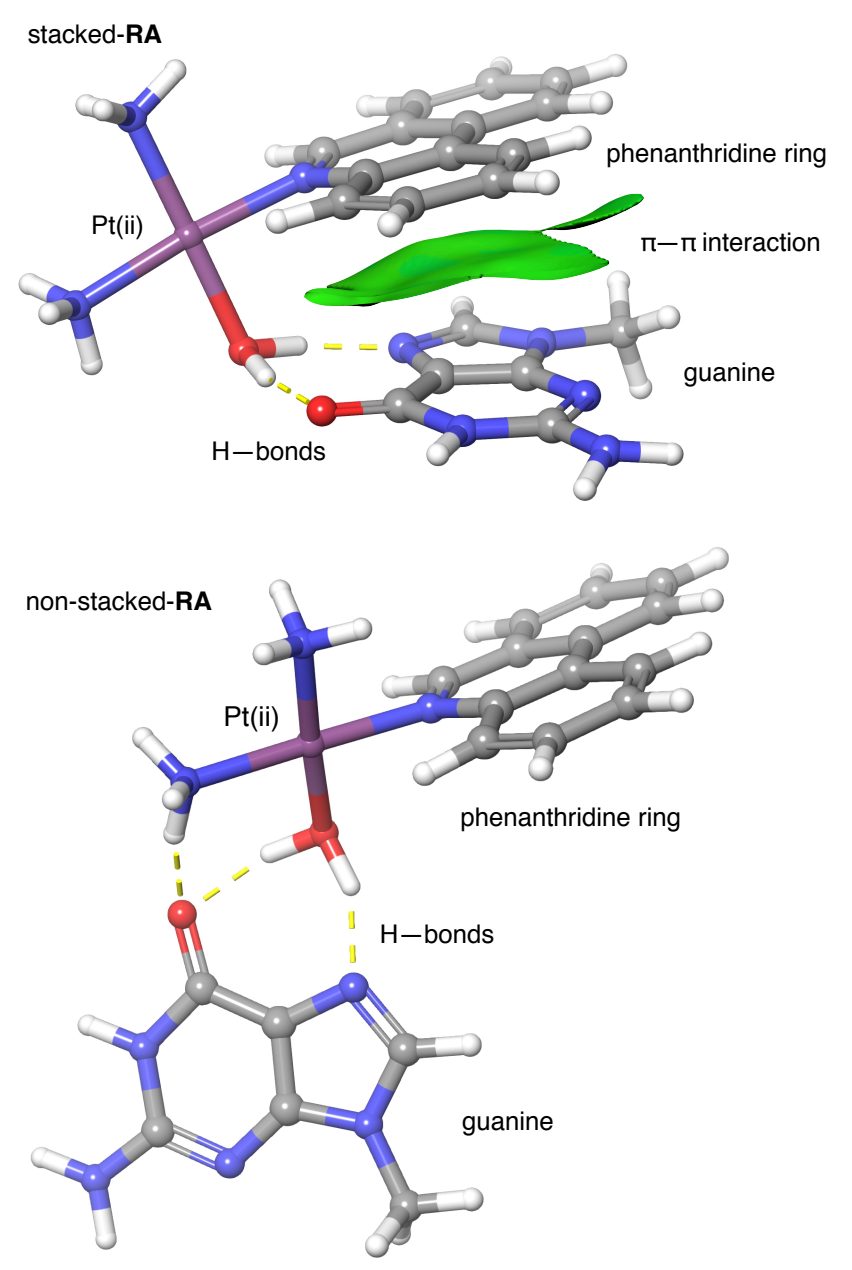

Figure 3. Reactive adducts (RA) during the attack of phenPt on the N7 guanine site. The stacked RA is compatible with the measured kinetics in free solution (upper panel), while the non-stacked form is the only structure possible in the reaction with DNA (lower panel). H-bonds are plotted as dashed yellow lines and $\pi-\pi$ stacking is shown as a green surface. Color scheme: gray, $\mathrm{C}$ atoms; blue, $\mathrm{N}$ atoms; red, $\mathrm{O}$ atoms; purple, $\mathrm{Pt}$ atoms; white, $\mathrm{H}$ atoms.

Our theoretical values correctly reproduce the attack on DNA by cisPt and the observed reaction trend of phenPt in the experiments (N7-guanine $>$ N7-adenine $>$ N1-adenine). However, the calculations with phenPt yield kinetic parameters that are several orders of magnitude larger than the reported values. ${ }^{7}$ Earlier experiments have shown that phenPt preferentially reacts at the N7 position of guanine with slow kinetics $\left(\Delta G^{\ddagger}=23.5 \mathrm{kcal} \mathrm{mol}^{-1}\right.$ and $\left.k=3.6 \times 10^{-5} \mathrm{~s}^{-1}\right)$. We therefore predict more rapid kinetics for phenPt than cisPt $\left(\Delta G^{\ddagger}=17.8 \mathrm{kcal} \mathrm{mol}^{-1}\right.$ and $k=$ $5.5 \times 10^{-1} \mathrm{~s}^{-1}$ ). Because good experimental/theoretical agreement was obtained at the hydrolysis stage, it is more likely that such a conflicting result arises from an inconsistency in the chemical model than from an error in the theory.
Figure 1 shows that phenPt binds covalently to a single guanine base. The phenanthridine ring is oriented outwards from the double helix and is not intercalated between two base pairs - that is, phenPt does not interact with the base pairs during the platination of DNA (see Figure $\mathrm{S} 7$ and ref. 5). However, if the reaction is designed using isolated bases, as in the high-performance liquid chromatography experiments from which the rate constants were obtained, ${ }^{7}$ phenPt can be assembled with guanine prior to attack by the metal. To confirm this hypothesis, the reaction was simulated by repositioning the bases opposite to the phenanthridine ring. Figure 3 shows that phenanthridine anchors the guanine base via $\pi-\pi$ stacking in the stacked RA, an interaction that is missing in the non-stacked RA. This additional contact can be seen as a green surface by computing the electron density and reduced density gradient as implemented in the NCIPlot code. ${ }^{23,24}$ The attack at the N7 position in guanine through a self-assembled reactant has to overcome an energy barrier $7 \mathrm{kcal} \mathrm{mol}^{-1}$ higher than in the nonstacked forms. The predicted activation energy is plotted in Figure 2 as dashed lines $\left(\Delta G^{\ddagger}=20.3 \mathrm{kcal} \mathrm{mol}^{-1}\right.$ and $k$ $=8.0 \times 10^{-3} \mathrm{~s}^{-1}$ ) and allows the experimental and theoretical results to be reconciled. The same effect is observed in the attack on adenine, with an increase of $c .5 \mathrm{kcal}$ $\mathrm{mol}^{-1}$. It should be stressed that the rate constant is very sensitive and a difference of $1 \mathrm{kcal} \mathrm{mol}^{-1}$ in the energetic barrier corresponds to one order of magnitude on the $k$ scale. Our computational strategy is therefore at the limit of chemical accuracy (with a difference of only $3 \mathrm{kcal}$ $\mathrm{mol}^{-1}$ ) if the reaction is modeled with self-assembled RA structures. ${ }^{25}$ More refined predictions can be produced using larger DNA fragments (e.g., a dodecamer structure), which will require the application of more elaborate computational approaches, such as quantum mechanics/molecular mechanics calculations. ${ }^{26,27}$ However, the use of isolated base pairs within the DFT framework is sufficient to provide convergence with the experiments and to predict reliable kinetic parameters.

The possible attack on sulfur-containing biomolecules was finally assessed by analyzing the reaction of the water-activated drug to a cysteine moiety. The reaction with proteins rich in this amino acid is a source of the nontargeted effects of these anticancer drugs, so the platination of cysteine provides a first model to predict unwanted reactions in healthy tissues. ${ }^{28}$ The optimized structures and reaction energy profiles (see SI) showed similar kinetics for the reactions of cisPt $\left(\Delta G^{\ddagger}=20.9 \mathrm{kcal} \mathrm{mol}^{-1}\right.$ and $\left.k=2.9 \times 10^{-3} \mathrm{~s}^{-1}\right)$ and phenPt $\left(\Delta G^{*}=19.0 \mathrm{kcal} \mathrm{mol}^{-1}\right.$ and $k=7.2 \times 10^{-2} \mathrm{~s}^{-1}$ ). The damage inflicted on proteins appears to be thermodynamically rather than kinetically controlled. cisPt $\left(\Delta G=-6.6 \mathrm{kcal} \mathrm{mol}^{-1}\right.$ and $K_{\text {eq }}=7.8 \times$ $\left.10^{4}\right)$ produces more stable damage at cysteine than phenPt $\left(\Delta G=2.7 \mathrm{kcal} \mathrm{mol}^{-1}\right.$ and $\left.K_{\text {eq }}=1.1 \times 10^{-2}\right)$. This mitigation of the attack on proteins confirms an additional advantage of monofunctional platinum drugs over classical bifunctional drugs. ${ }^{29}$ 
Our results outline the biological mechanisms of phenPt, one of the most promising monofunctional platinum drugs. The simulations are fully consistent with the available experimental data, with the key exception of the kinetics associated with the attack on the DNA bases. In contrast with the results obtained in solution, our calculations suggest that the attack of phenPt on DNA is even faster than that of cisPt and that hydrolysis is the ratelimiting step in both reactions. The source of this inconsistency may be explained by stacking of the phenanthridine ring that assembles the phenPt and bases prior to the platination reaction. The stacked reactant in Figure 3, which is accessible in free solution but inaccessible in the double helix, slows the kinetics down by several orders of magnitude.

Although monofunctional drugs may improve the response of patients to chemotherapy, attempts to optimize the parent phenPt molecule have so far been unsuccessful. We hope that this report of self-assembled reactants will provide experimentalists with new lines of investigation in the design of improved anticancer drugs.

\section{ASSOCIATED CONTENT}

The Supporting Information is available free of charge on the ACS Publications website: (i) computational details; (ii) optimized chemical structures; (iii) geometrical parameters; and (iv) the imaginary frequencies of all located transition states.

\section{AUTHOR INFORMATION}

\section{Corresponding Authors}

andrea.melchior@uniud.it; jpceron@ucam.edu

\section{ACKNOWLEDGMENTS}

This research used resources from (i) the local Galileo cluster installed at UCAM, Spain; (ii) the Plataforma Andaluza de Bioinformática at the Universidad of Málaga, Spain; and (iii) "Piano Strategico" of the University of Udine for funding the local high-performance computing resources.

\section{REFERENCES}

1. Rosenberg, B.; Van Camp, L.; Krigas, T. Inhibition of Cell Division in Escherichia Coli by Electrolysis Products from a Platinum Electrode. Nature 1965, 205, 698-699.

2. Kelland, L. The resurgence of platinum-based cancer chemotherapy. Nat. Rev. Cancer. 2007, 7, 573-584.

3. Park, G. Y.; Wilson, J. J.; Song, Y.; Lippard, S. J. Phenanthriplatin, a Monofunctional DNA-binding Platinum Anticancer Drug Candidate with Unusual Potency and Cellular Activity Profile. PNAS 2012, 109, 11987-11992.

4. Jung, Y.; Lippard, S. J. Direct Cellular Responses to Platinum-Induced DNA Damage. Chem. Rev. 2007, 107, 13871407.

5. Gregory, M. T.; Park, G. Y.; Johnstone, T. C.; Lee, Y. S.; Yang, W.; Lippard, S. J. Structural and Mechanistic Studies of Polymerase $\eta$ Bypass of Phenanthriplatin DNA Damage. PNAS 2014, 111, 9133-9138.

6. Casini, A.; Reedijk, J. Interactions of Anticancer Pt Compounds with Proteins: an Overlooked Topic in Medicinal Inorganic Chemistry? Chem. Sci. 2012, 3, 3135.
7. Riddell, I. A.; Johnstone, T. C.; Park, G. Y.; Lippard, S. J. Nucleotide Binding Preference of the Monofunctional Platinum Anticancer-Agent Phenanthriplatin. Chem. Eur. J. 2016, 22, 7574-7581.

8. Alberto, M. E.; Lucas, M. F. A.; Pavelka, M.; Russo, N. The Second-Generation Anti-Cancer Drug Nedaplatin: A Theoretical Investigation on the Hydrolysis Mechanism. J. Phys. Chem. B 2009, 113, 14473-14479.

9. Cerón-Carrasco, J. P.; Jacquemin, D.; Cauët, E. Cisplatin Cytotoxicity: A Theoretical Study of Induced Mutations. Phys. Chem. Chem. Phys. 2012, 14, 12457-12464.

10. Melchior, A.; Martínez, J. M.; Pappalardo, R. R.; Sánchez Marcos, E. Hydration of Cisplatin Studied by an Effective Ab Initio Pair Potential Including Solute Solvent Polarization. J. Chem.Theory Comput. 2013, 9, 4562-4573.

11. Melchior, A.; Tolazzi, M.; Martínez, J. M.; Pappalardo, R. R.; Sánchez Marcos, E. Hydration of Two Cisplatin AquaDerivatives Studied by Quantum Mechanics and Molecular Dynamics Simulations. J.Chem. Theory Comput. 2015, 11, 1735-44.

12. Ritacco, I.; Al Assy, M.; Abd El-Rahman, M. K.; Fahmy, S. A.; Russo, N.; Shoeib, T.; Sicilia, E. Hydrolysis in Acidic Environment and Degradation of Satraplatin: A Joint Experimental and Theoretical Investigation. Inorg. Chem. 2017, $56,6013-6026$

13. Xu, Z.; Zhou, L. A DFT study of a novel oxime anticancer trans platinum complex: Monofunctional and bifunctional binding to purine bases. Int. J. Quantum Chem., 2011, 111: 1907-1920.

14. Grimme, S.; Ehrlich, S.; Goerigk, L. Effect of the Damping Function in Dispersion Corrected Density Functional Theory. J. Comput. Chem. 2011, 32, 1456-1465.

15. Frisch, M. J.; Trucks, G. W.; Schlegel, H. B.; Scuseria, G. E.; Robb, M. A.; Cheeseman, J. R.; Scalmani, G.; Barone, V.; Petersson, G. A.; Nakatsuji, H. et al. Gaussian16 Rev. A.03. 2016; Gaussian Inc. Wallingford $C T$.

16. Johnstone, T. C.; Lippard, S. J. The Chiral Potential of Phenanthriplatin and Its Influence on Guanine Binding. $J$. Am Chem. Soc. 2014, 136, 2126-2134.

17. Hindmarsch, K.; House, D. A.; Turnbull, M. M. The hydrolysis Products of Cis-diamminedichloroplatinum(II) 9. Chloride and Bromide Anation Kinetics for Some [PtII(N) $\left.)_{2}\left(\mathrm{OH}_{2}\right)_{2}\right]^{2+}$ Complexes and the Structures of $\left[\mathrm{PtIVBr}_{4}(\mathrm{~N})_{2}\right]\left((\mathrm{N})_{2}=\mathrm{en}, \mathrm{tn}\right)^{1}$. Inorganica Chim. Acta 1997, $257,11-18$.

18. Lau, J. K. C.; Deubel, D. V. Hydrolysis of the Anticancer Drug Cisplatin: Pitfalls in the Interpretation of Quantum Chemical Calculations. J. Chem. Theory Comput. 2006, 2 , 103-106.

19. Melchior, A.; Sánchez Marcos, E.; R. Pappalardo, R.; Martínez, J. M. Comparative Study of the Hydrolysis of a Third- and a First-generation Platinum Anticancer Complexes. Theor. Chem. Acc. 2011, 128, 627-638.

20. Graziani, V.; Coletti, C.; Marrone, A.; Re, N. Activation and Reactivity of a Bispidine Analogue of Cisplatin: A Theoretical Investigation. J. Phys. Chem. A 2016, 120, 5175-5186.

21. Raber, J.; Zhu, C.; Eriksson, L. A. Theoretical Study of Cisplatin Binding to DNA: The Importance of Initial Complex Stabilization. J. Phys. Chem. B 2005, 109, 11006- 11015.

22. Xu, Z.; Zhou, L. A DFT Study of a Novel Oxime Anticancer Trans Platinum Complex: Monofunctional and Bifunctional Binding to Purine Bases. Int. J. Quantum Chem. 2011, 111, 1907-1920.

23. Johnson, E.R.; Keinan, S.; Mori-Sánchez, P.; ContrerasGarcía, J.; Cohen, A.J.; Yang, W. Revealing Noncovalent Interactions. J. Am. Chem. Soc. 2010, 132, 6498-6506.

24. Contreras-García, J.; Johnson, E.R.; Keinan, S.; Chaudret, R.; Piquemal, J.P.; Beratan, D.N.; Yang, W. NCIPLOT: A Program for Plotting Noncovalent Interaction Regions. $J$. Chem. Theory Comput. 2011, 7, 625-632. 
25. Piccini, G.; Aelssio M..; Sauer, J. Ab Initio Calculation of Rate Constants for Molecule-Surface Reactions with Chemical Accuracy. Angew. Chem. Int. Ed. 2016, 55, 5235-5237.

26. Garrec, J.; Patel, C.; Rothlisberger, U.; Dumont, E. Insight into Intrastrand Cross-Link Lesions of DNA from QM/MM Molecular Dynamics Simulations. J. Am. Chem. Soc. 2012, 134, 2111-2119.

27. Galano A.; Alvarez-Idaboy, J.R. On the Evolution of OneElectron-Oxidized Deoxyguanosine in Damaged DNA Under Physiological Conditions: a DFT and ONIOM Study on Proton Transfer and Equilibrium. Phys. Chem. Chem. Phys. 2012, 14, 12476-12484.

28. Zimmermann, T.; Chval, Z.; Burda, J. V. Cisplatin Interaction with Cysteine and Methionine in Aqueous Solution: Computational DFT/PCM Study. J. Phys. Chem. B 2009, 113, 3139-3150.
29. Zhou, W.; Almeqdadi, M.; Xifaras, M.E.; Riddell, I.A.; Yilmaz, O.H.; Lippard, S.J. The effect of geometric isomerism on the anticancer activity of the monofunctional platinum complex trans-[Pt $\left(\mathrm{NH}_{3}\right)_{2}$ (phenanthridine) $\left.\mathrm{Cl}\right] \mathrm{NO}_{3}$. Chem. Commun. 2018, 54, 2788-2791. 
Table of Contents

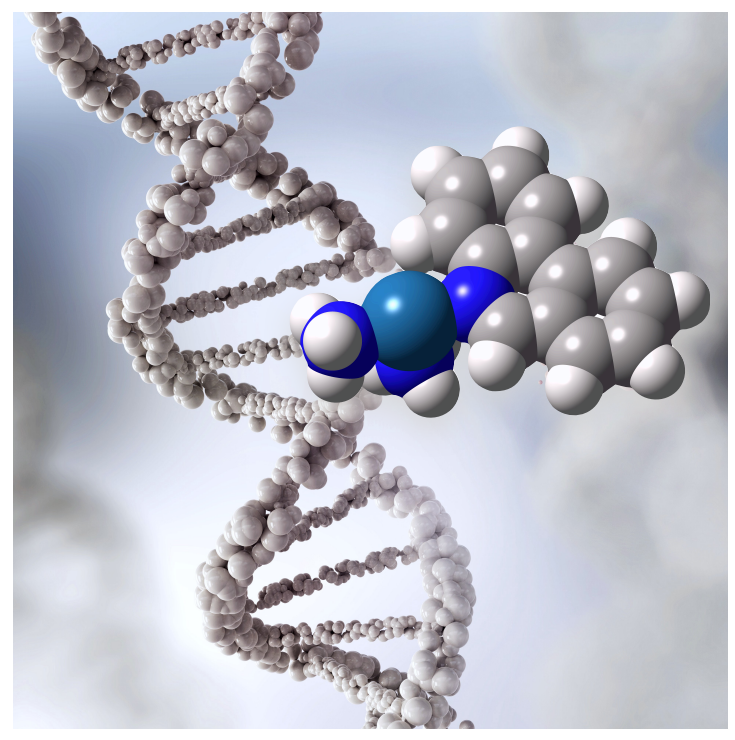

